Identification and Preliminary Structure-Activity Relationships of Dietary Flavonoids as

\title{
Human Macrophage Migration Inhibitory Factor (MIF) Inhibitors
}

Liu Yang ${ }^{1}$, Deyin Guo ${ }^{1,2^{*}}$, and Chengpeng Fan ${ }^{1 *}$

${ }^{1}$ School of Basic Medical Sciences, Wuhan University, Wuhan 430072, P. R. China

${ }^{2}$ Center for Infection \& Immunity Study (CIIS), School of Medicine, Sun Yat-sen University, Shenzhen 518107, P. R. China.

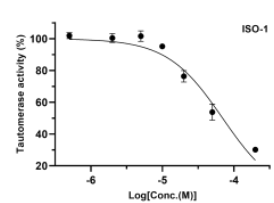

A
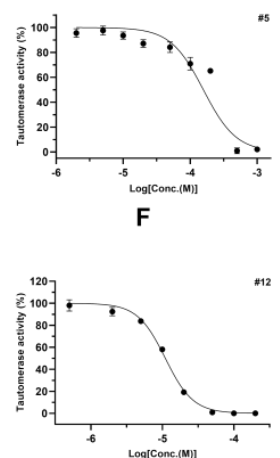

K
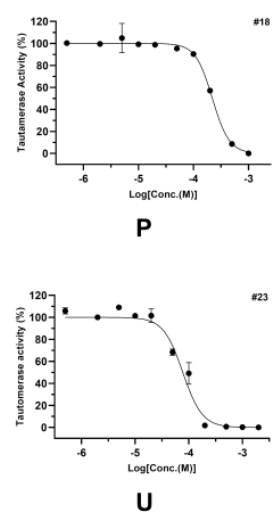

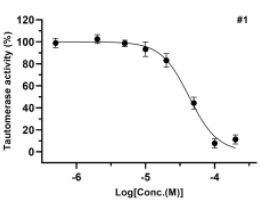

B
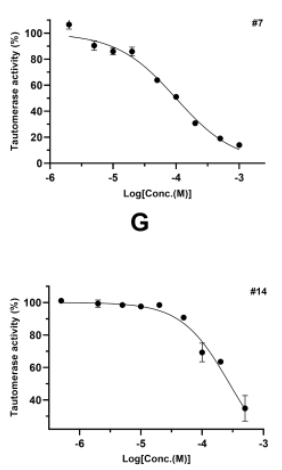

L
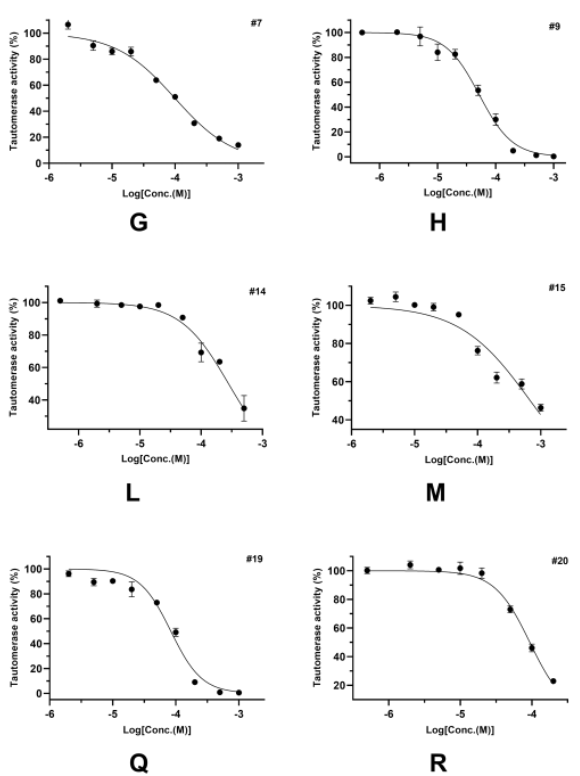

M
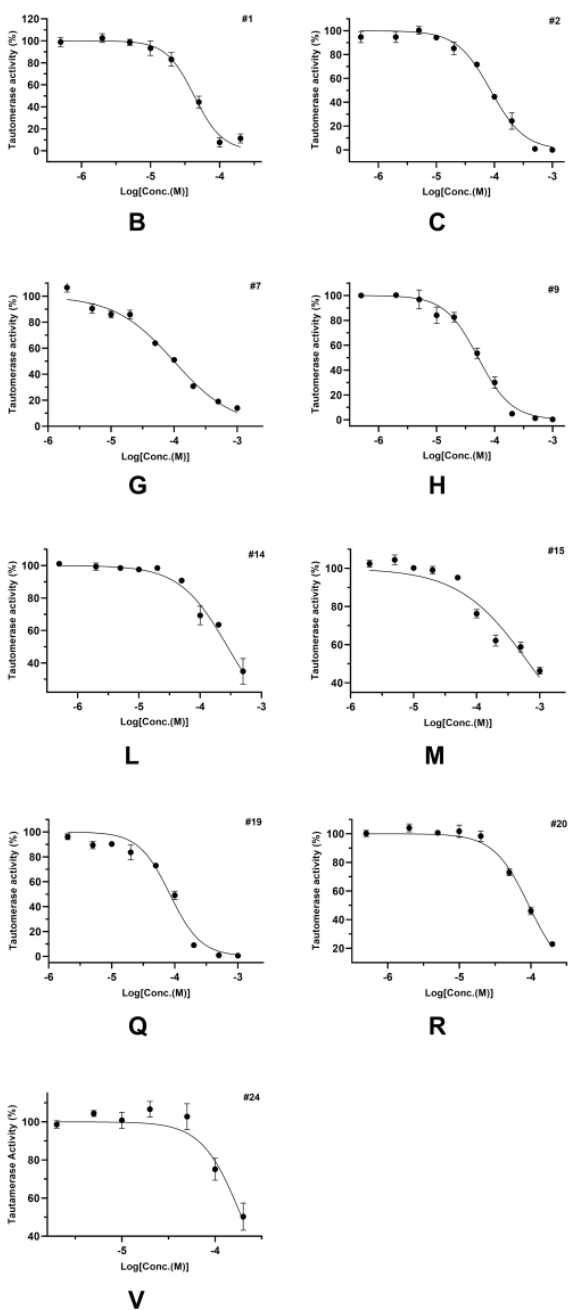
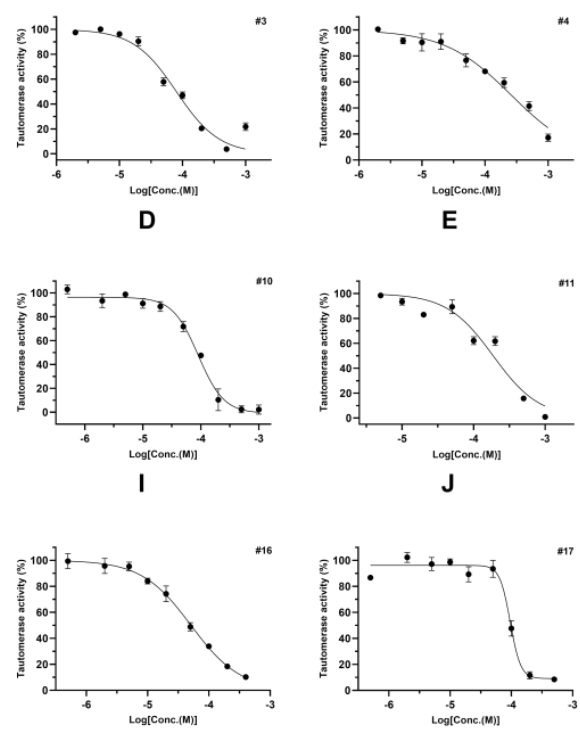

N

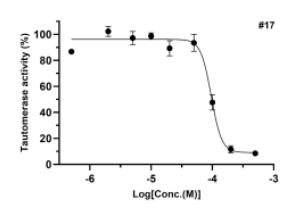

o
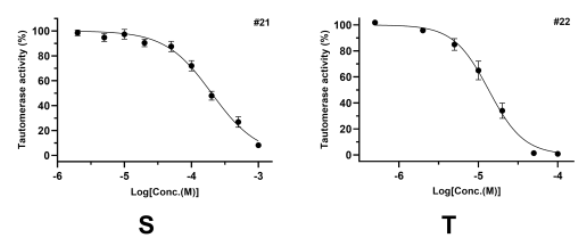

Figure S1. $\mathrm{IC}_{50}$ determination of selected dietary flavonoids against MIF tautomerase activity.

The enzyme activity was assessed by measuring the increase in absorbance at $306 \mathrm{~nm}$ at $8 \mathrm{~s}$ 
intervals over $90 \mathrm{~s}$ immediately after adding the substrate 4-HPP $(2 \mathrm{mM})$. The $\mathrm{IC}_{50}$ value of each compound (A-V) was obtained by dose-response curves fitting to the nonlinear regression analysis using GraphPad Prism version 8.30. Each graph represents mean \pm SEM; $n=3$.

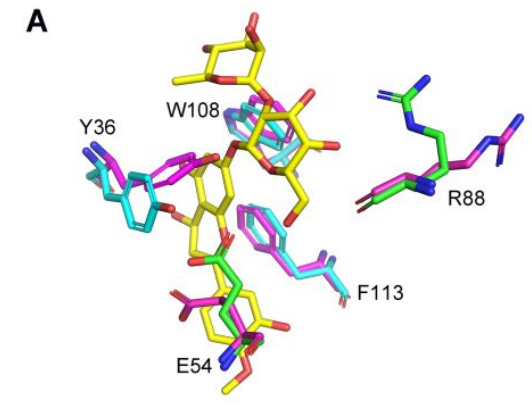

B

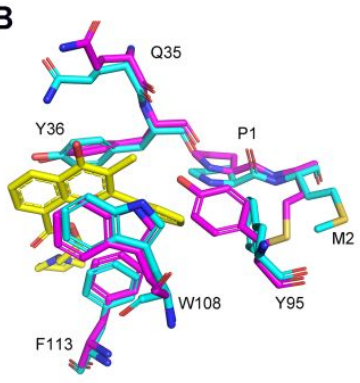

D

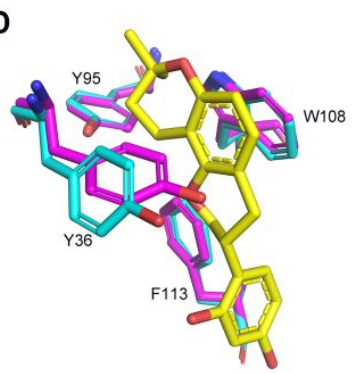

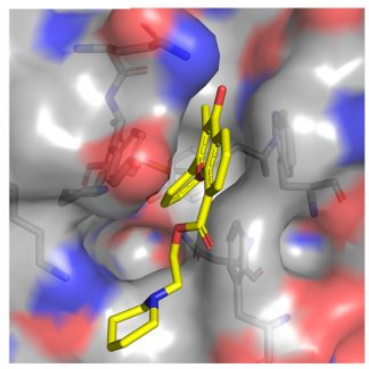

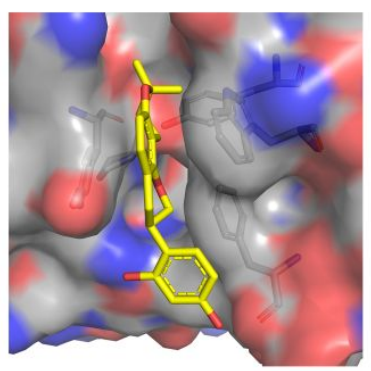

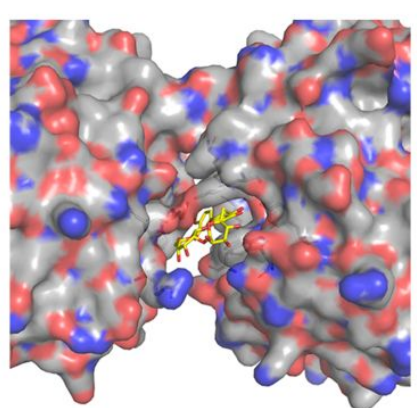

C

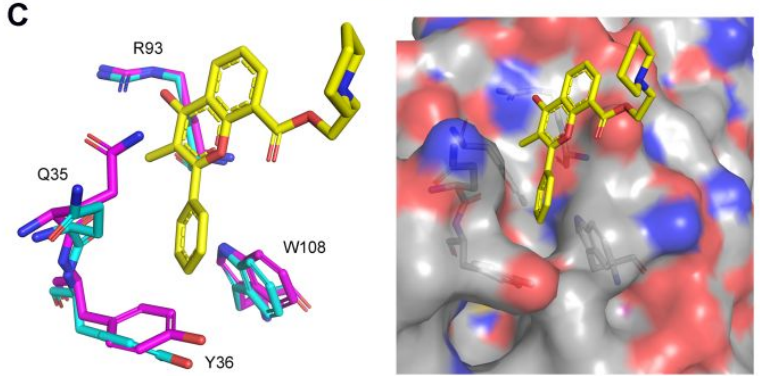

E

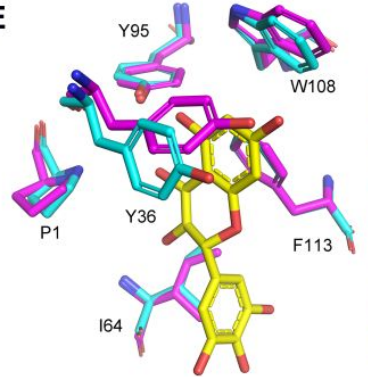

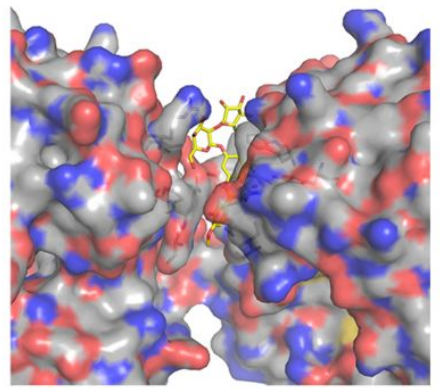

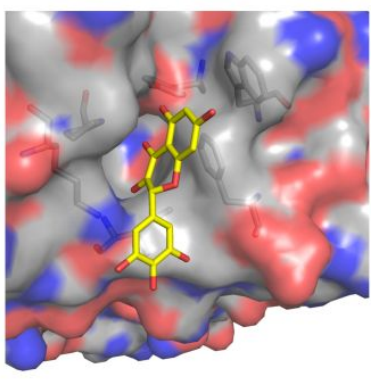

Figure S2. Section views of flavonoids $(\mathbf{1 4}, \mathbf{1 8}, \mathbf{2 3}$, and 24) binding and the conformational change of MIF. Structure superposition of 14 (A), 18 (E), 23 (D), or 24 (B, C) bound to MIF (cyan) with the apo-MIF (PDB entry 1MIF, magenta) are depicted. Ligands are shown as yellow sticks. The solvent accessible surfaces of ligand-binding pockets are indicated with the electrostatic potentials. 